\title{
ON THE INVARIANCE OF CERTAIN CLASSES OF FUNCTIONS UNDER COMPOSITION
}

\author{
MILTON CHAIKA ${ }^{1}$ AND DANIEL WATERMAN
}

\begin{abstract}
Certain classes of functions are mapped into themselves by any change of variable. For some classes of this type which are of interest in the study of Fourier series, it is shown that the necessary and sufficient condition that $g \circ f$ be in the class for each $f$ of that class is that $g \in \operatorname{Lip} 1$.
\end{abstract}

1. The class of functions of bounded variation on an interval has the property of invariance under a change of variable, i.e., if $f \in B V$ and $h$ is a homeomorphism of the interval onto itself, then $f \circ h \in B V$. This is a property of several classes of interest in the study of Fourier series. Among these are the class $G W$ of functions with only simple discontinuites which have a convergent Fourier series after every change of variable, and the class $U G W$ defined analogously with respect to uniform convergence.

Another such class is the class $H B V$, the functions of harmonic bounded variation, defined as follows. If $I=[a, b]$, write $f(I)=f(b)-f(a)$. We say that $f \in H B V$ if $\sum_{1}^{\infty} f\left(I_{n}\right) / n<\infty$ for every collection $\left\{I_{n}\right\}$ of nonoverlapping intervals.

It is known that ([3], [6]) $G W \supseteq H B V$, and that ([1], [6]) $G W_{\text {(cont.) } \supsetneq}$ $U G W \supseteq H B V_{\text {(cont.). }}$.

Here we are concerned with the question of which functions have the property that they leave these classes invariant under composition on the left. We assume throughout that the domain of such a function $g$ is an interval and $g \circ f$ is well defined. We have the following

THEOREM. $g \circ f$ is in one of the classes $G W, U G W$, or $H B V$ for each $f$ of that class whose range is in the domain of $g$ if and only if $g \in \operatorname{Lip} 1$.

2. The necessary and sufficient conditions for a function to belong to $G W$ or $U G W$ are phrased in terms of "systems of intervals" at a point. For each $n$, let $I_{n m}, m=1,2, \cdots, k_{n}$, be disjoint closed intervals such

Received by the editors October 20, 1972.

AMS (MOS) subject classifications (1970). Primary 42A20, 26A45, 26A16.

Key words and phrases. Lipschitz classes, compositions, convergence of Fourier series, generalized bounded variation.

${ }^{1}$ Partially supported by NSF Grant GP-19653A-1. 
that $I_{n, m-1}$ is to the left of $I_{n m}$. Let there be a real $x$ such that for every $\delta>0$ there is an $N$ such that $I_{n m} \subset(x, x+\delta)$ whenever $n>N$. Then the collection $\mathfrak{I}=\left\{I_{n m}: n=1,2, \cdots ; m=1,2, \cdots, k_{n}\right\}$ is called a right system of intervals at $x$. A left system is defined similarly. Goffman and Waterman [4] also required that $\lim _{n \rightarrow \infty} k_{n}=\infty$ and $\lim _{n \rightarrow \infty} n^{-1} k_{n}=0$. Baernstein and Waterman [1] showed this requirement to be redundant.

Goffman and Waterman [4] showed that $f \in G W$ if and only if

$$
\lim _{n \rightarrow \infty} \sum_{1}^{k_{n}} f\left(I_{n m}\right) / m=0
$$

for every system $\mathfrak{I}$. Baernstein and Waterman [1] showed that a continuous $f \in U G W$ if and only if both (1) and

$$
\lim _{n \rightarrow \infty} \sum_{1}^{k_{n}} f\left(I_{n m}\right) /\left(k_{n}+m-1\right)=0
$$

hold for every system $\mathfrak{I}$.

It is clear that if we add to (1) and (2) the requirement that for each $n$ the sign of $f\left(I_{n m}\right)$ be constant, we obtain equivalent conditions and, therefore, if we replace $f\left(I_{n m}\right)$ in these conditions by $\left|f\left(I_{n m}\right)\right|$, we obtain still another set of equivalent conditions.

Since $g \in$ Lip 1 implies that there is a $C>0$ such that $|g \circ f(I)|<$ $C|f(I)|$ for every interval $I$, it is obvious, in view of the above remarks, that composition on the left with functions of class Lip 1 preserves $G W$, $U G W$, and $H B V$.

We will now show that continuity of $g$ is a necessary condition for preservation of these classes. One way in which this might be done is to adapt the classical constructions of a function whose Fourier series diverges at a point to fit our situation. It is far simpler, however, to use the characterizations of these classes given above.

Without loss of generality, we may suppose that $g(0)=0$ and $g\left(t_{n}\right) \geqq 1$, where $t_{n} \searrow 0$ and $\sum_{1}^{\infty} t_{n}<\pi$. Let $\left\{I_{n}\right\}$ be a sequence of disjoint closed intervals in $(0, \pi)$ which converges downward to the origin. Define $f$ to be $t_{n}$ on $I_{n}$ for each $n$ and to be zero at $-\pi, 0, \pi$, and at the midpoint of the interval between $I_{n}$ and $I_{n+1}$ for each $n$. On each of the component intervals of the set in $[-\pi, \pi]$ on which $f$ is not yet defined, let $f$ be linear and such that the resulting function is continuous on $[-\pi, \pi]$. Clearly $g \circ f$ is not in the class $G W$ and, therefore, not in either $U G W$ or $H B V$. However, $f$ is a continuous function of bounded variation and is, therefore, in all three of these classes.

3. We will now show that the condition on $g$ is necessary. We require certain information for this demonstration. 
If $f$ is a continuous function on an interval, the Banach indicatrix of $f, n(y ; f)$ is defined to be the cardinality of $\{x: f(x)=y\}$ if this set is finite and $+\infty$ otherwise. The class $G S$ of functions for which $\int \log n(y ; f) d y<$ $\infty$ was introduced by Beurling. Integration here is over the range of $f$. It was shown by Garsia and Sawyer [2] that the functions of class $G S$ have uniformly convergent Fourier series. Clearly $U G W \supseteq G S$. It is also true ([3], [6]) that $H B V_{\text {(cont.) }} \supseteq G S$.

It is interesting to note that these inclusions are proper. This follows from a result of Kiselman [5] to the effect that $G S$ is not closed under addition. Clearly the other classes are linear spaces and, indeed, $H B V$ and $H B V_{\text {(cont.) }}$ are Banach spaces [6].

We shall show that if $g \notin \operatorname{Lip} 1$, then there is a function $f \in G S$ such that $g \circ f \notin G W$.

Since we may assume that $g$ is continuous, it is easy to see that, without loss of generality, we may suppose that there is a sequence of disjoint closed intervals $J_{n}$ in $(0,1)$ with endpoints $\searrow 0$ and a sequence of real numbers $c_{n} \rightarrow \infty$ such that $\sum_{1}^{\infty} 1 / c_{n}<\infty$ and $\left|J_{n}\right| c_{n}<g\left(J_{n}\right)$. Clearly $g\left(J_{n}\right) \rightarrow 0$. Let

$$
k_{n}=\left[\exp \left(1 / c_{n}\left|J_{n}\right|\right)\right]+1 .
$$

Then $k_{n} \rightarrow \infty$ and

$$
g\left(J_{n}\right) \log k_{n} \geqq g\left(J_{n}\right) / c_{n}\left|J_{n}\right| \geqq 1 .
$$

Also, for some $C>0$,

$$
\sum_{1}^{\infty}\left|J_{n}\right| \log k_{n}<C \sum_{1}^{\infty} 1 / c_{n}<\infty .
$$

For each $n=1,2, \cdots$, let $I_{n 1}, I_{n 2}, \cdots, I_{n k_{n}}$ be a collection of disjoint closed intervals in $\left(\pi / 2^{n}, \pi / 2^{n-1}\right)$ with $I_{n m}$ to the left of $I_{n, m+1}$ for each $m$. Let $f$ be defined on each $I_{n m}, m=1, \cdots, k_{n} ; n=1,2, \cdots$, to be the increasing linear map of $I_{n m}$ onto $J_{n}$. Let $f(-\pi)=f(0)=f(\pi)=0$. Define $f$ to be linear on each of the component intervals of the remainder of $[-\pi, \pi]$ and continuous on $[-\pi, \pi]$. Clearly

$$
\int \log n(y ; f) d y \leqq \log 2+\sum_{1}^{\infty}\left|J_{n}\right| \log 2 k_{n}<\infty,
$$

and so $f \in G S$. But

$$
\sum_{m=1}^{k_{n}} g \circ f\left(I_{n m}\right) / m=g\left(J_{n}\right) \sum_{1}^{k_{n}} 1 / m>g\left(J_{n}\right) \log k_{n} \geqq 1 .
$$

Hence $g \circ f \notin G W$. 


\section{REFERENCES}

1. A. Baernstein and D. Waterman, Functions whose Fourier series converge uniformly for every change of variable, Indiana Univ. Math. J. (to appear).

2. A. M. Garsia and S. Sawyer, On some classes of continuous functions with convergent Fourier series, J. Math. Mech. 13 (1964), 589-601. MR 33 \#7777.

3. C. Goffman, Everywhere convergence of Fourier series, Indiana Univ. Math. J. 20 (1970/71), 107-112. MR 42 \#4941.

4. C. Goffman and D. Waterman, Functions whose Fourier series converge for every change of variable, Proc. Amer. Math. Soc. 19 (1968), 80-86. MR 36 \#4245.

5. C. O. Kiselman, On the Garsia-Sawyer condition for uniform convergence of Fourier series, Uppsala University, Dept. of Mathematics, Report No. 25, 1971.

6. D. Waterman, On convergence of Fourier series of functions of generalized bounded variation, Studia Math. 44 (1972), 107-117.

Department of Mathematics, Detroit Institute of Technology, Detroit, MiCHIGAN 48221

Department of Mathematics, Syracuse University, Syracuse, New York 13210 